Thomas Moore et A. de Vigny

Author(s): Fernand Baldensperger

Source: The Modern Language Review, Vol. 1, No. 4 (Jul., 1906), pp. 290-301

Published by: Modern Humanities Research Association

Stable URL: http://www.jstor.org/stable/3713452

Accessed: 27-02-2016 18:31 UTC

Your use of the JSTOR archive indicates your acceptance of the Terms \& Conditions of Use, available at http://www.jstor.org/page/ info/about/policies/terms.jsp

JSTOR is a not-for-profit service that helps scholars, researchers, and students discover, use, and build upon a wide range of content in a trusted digital archive. We use information technology and tools to increase productivity and facilitate new forms of scholarship. For more information about JSTOR, please contact support@jstor.org. 


\section{THOMAS MOORE ET A. DE VIGNY.}

Deux articles récents, l'un de M. Ernest Dupuy sur les Origines littéraires d'A. de Vigny ${ }^{1}$, l'autre de M. Schultz-Gora sur Eloa ${ }^{2}$, ont insisté sur les emprunts ou les réminiscences qui rattachent ce 'mystère' à d'autres œuvres, surtout étrangères. Ils sont d'accord pour réduire au minimum l'influence qu'a pu exercer Moore sur Vigny, et le poème des Amours des Anges leur semble devoir être rayé du nombre des ouvrages dont procéderait à quelque degré la Sceur des Anges. Ils s'écartent en ceci de l'opinion de Sainte-Beuve qui mettait en 1835 'Thomas Moore lui-même' parmi les 'sources extérieures du talent poétique de M. de Vigny, si on les recherche bien".' Jès la publication d'Eloa, Henri de Latouche, lié avec Vigny, avait, dans le Mercure du $X I X^{e}$ Siècle, indiqué sans s'y arrêter que 'l'invention de cette fable a quelque analogie avec tel poème de Moore, ou de Byron'; un rédacteur du Globe, Ch. Magnin, notait le 21 octobre 1829 'qu'auprès d'Eloa, les A'nours des Anges, de Thomas Moore, ne sont qu'une mesquine et coquette conception, un feu follet sans consistance et sans portée.' Même en supposant un classement aussi inégal des deux ouvrages, le rapprochement paraissait s'imposer: on le rencontre encore sous la plume d'un critique aussi informé qu'Emile Montégut: 'Eloa a son origine dans les Amour's des Anges de Moore,' écrit-il dans la Revue des Deux Mondes du $1^{\text {er }}$ mars $1867^{4}$. Il est permis enfin de retrouver un souvenir déguisé de cette association presque convenue de deux œuvres séraphiques dans l'allusion faite à la fois par Balzac aux Amours des Anges et à une série de créations plus ou moins analogues, lorsqu'il parle-assez à l'improviste-du 'poème caressé par tant de poètes, par Moore, par lord Byron, par Mathurin, par Canalis (un démon possédant

1 Revue d'histoire littéraire de la France, x. (1903), p. 373. Reproduit en volume dans la Jeunesse des Romantiques, Paris, 1905.

2 Zeitschrift für französische Sprache und Litteratur, xxvir. (1904), p. 278. p. 411).

3 Portraits contemporains, t. II. p. 62 . Il y revient en 1864 (Nouveaux Lundis, t. vi.

4 Daus Nos morts contemporains, $1^{\mathrm{e}}$ série, p. 344. 
un ange attiré dans son enfer pour le rafraîchir d'une rosée dérobée au paradis)1.' Quelle est en réalité la nature de ces rapports ou de cette dépendance?

Pour le premier romantisme, celui de 1820, qui n'avait pas encore fait sa révolution en matière de langue et de style et qui mettait presque tout son effort à créer une sorte de littérature transcendentale, le poète du Paradis et la Péri n'avait pu manquer d'apparaître comme un auxiliaire précieux. De fait, on le tient en singulière estime, et c'est à peine si W. Scott et Byron font tort à sa renommée. 'On dirait, écrivent les Annales de la littérature et des arts", que ces trois génies se sont divisé entre eux toute la création. W. Scott s'est emparé de la terre; lord Byron semble s'être précipité dans les sombres abîmes; le domaine des cieux est échu à Th. Moore. Il semble, en quelque sorte, initier l'homme aux sublimes mystères de la divinité.' C'est à propos des Amours des Anges que l'organe attitré de la Société des BonnesLettres procède à cette répartition de l'univers; et ce poème semblait en effet mettre le sceau à la renommée de Moore considéré comme le peintre des régions supérieures. Mais il n'avait pas attendu jusque$l^{3}{ }^{3}$ pour exercer une influence dont Fontaney, Guttinguer, Gérard de Nerval offrent mainte trace: Berlioz et Th. Gautier à leur tour représenteront, dans la troupe des admirateurs français du poète irlandais, comme un second ban, qui l'aimera pour des qualités différentes.

Alfred de Vigny a dû de très bonne heure être mis an fait de l'œuvre de Moore par son parent Bruguière de Sorsum : celui-ci publia en effet dès 1820, dans le Lycée français, un long article sur LallaRookh, où il s'attardait surtout à l'ingénieux épisode du Paradis et la Péri $i^{5}$. On voudrait pouvoir découvrir le nom du jeune poète aristocrate lui-même sous l'initiale V. qui signe un article du Conservateur littéraire, en juin 1820, consacré lui aussi à Lalla Rookh ${ }^{6}$. L'auteur remarquait que 'le style est ce qui prête le plus à l'éloge et à la critique' dans ce poème, mais il trouvait les défauts 'bien rachetés

\footnotetext{
1 La Dernière Incarnation de Vautrin [décembre 1847]. Ed. des Euvres complètes, Paris, 1860, t. xIx. p. 19.

2 1823 , tome xI. p. 95.

3 Une des premières mentions de Moore semble se trouver dans un article du Lycée français, 1819 , tome I. p. 131.

${ }^{4}$ C'est surtout autour de l'Epicurien que se rassemblent, après 1827 , ces nouvelles admirations.

${ }_{5}^{5}$ Lycée français, 1820, t. IIr. pp. 319, 363, 409 (à propos de la traduction Pichot).

${ }^{6}$ Conservateur littéraire, $2^{\mathrm{e}}$ année, livraison xv. p. 180 . Sainte-Beuve l'attribue à Hugo, semble-t-il. Selon M. Dupuy (Jeunesse des Romantiques, p. 231), le premier article de Vigny dans ce périodique serait celui qu'il consacrait à lord Byron sous cette même rubrique de Littérature anglaise, mais en décembre 1820. La Péri, 'venant du soleil,' paraît déjà dans Hélèna.
} 
par la variété des figures, léclat du coloris, la grâce ou l'énergie des peintures et cette vérité de teinte locale qui répand, sur les imperfections mêmes, une sorte de charme magique.' Surtout il observait que 'les ouvrages de Th. Moore, qui ont plu généralement, choqueront toutefois le goût de quelques champions du classique sans qu'ils puissent motiver leur sévérité. La poésie romantique, par ses formes vagues et indécises, échappe à la critique, semblable à ces hôtes fantastiques de l'Elysée païen, qui frappaient la vue et se dérobaient à la main qui les voulait saisir.' N'était-ce pas définir dans un sens bien conforme à la tendance du premier Cénacle les particularités preférées du poète irlandais?

Vigny allait subir, à l'égard de Moore, l'effet d'un autre prestige, et l'un de ceux qu'un apprenti de lettres éprouve presque infailliblement. De la fin de 1819 au printemps de 1822, l'ami et le confident de lord Byron, l'hôte favori de l'aristocratie libérale fit à Paris plusieurs séjours dont le jeune lieutenant de la garde royale a été certainement plus qu'informé. Le Journal de Moore mentionne ${ }^{1}$ un grand nombre de visites, de soirées, de rencontres mondaines qui mettent le poète étranger en présence de Mmes de Flahaut, de Sainte-Aulaire, de Barante, de Dolomieu, et surtout de la duchesse de Broglie, la fille de Mme de Staël, que Moore retrouvait à Paris après l'avoir déjà connue en Angleterre avec sa mère ${ }^{2}$, pendant les derniers mois de l'Empire. On aimerait savoir les noms des convives que $\mathrm{Mme}$ de Broglie fit rencontrer ì Moore le 19 janvier 1820, et l'on est tenté de s'impatienter de la mémoire indifférente, dédaigneuse ou courte de l'illustre étranger, qui note dans son journal: 'Treize personnes outre moi, qui étais le seul Anglais présent. Il y avait là quelques hommes dont on vante le talent, mais je ne me rappelle pas bien leurs noms. Il discutèrent littérature anglaise aussi couramment que s'ils connaissaient rien à l'affaire....' Il est certain, ̀̀ tout le moins, que Lamartine, qui est en relations avec Mme de Broglie dès $1819^{3}$, dont Moore traduit en 1820 quelques vers pour l'Edinburgh Review, a été admis à l'honneur d'approcher l'auteur de Lalla Rookh chez Mme de Broglie. Et il associe d'une façon si irrésistible ce souvenir à celui de Vigny, qu'il est permis de présumer que son émule en poésie reçut dès cette époque le contre-coup de l'émotion ressentie par les privilégiés qu'invita le duc

1 Memoirs, Journal and Correspondence of Th. Moore, London, 1853, vol. III.

2 Il la retrouve chez Mme de Flahaut le 19 décembre 1819.

3 Cf. les Confidences, éd. Hachette, livre xi. p. 304. Mme de Broglie parle de Lamartine à son amie Mme Anisson du Perron dans une lett're du 11 avril 1820 (Lettres, publiées par son fils, Paris, 1896, p. 42). 
de Broglie. Après avoir rappelé ${ }^{1}$ qu'il avait été lié avec Vigny 'depuis le jour où il répandit son nom dans le monde,' et avoir indiqué l'inspiration byronienne de Dolorida, Lamartine continue: 'Une autre imitation plus étudiée tentait déjà l'âme douce et tendre de Vigny. Thomas Moore, Irlandais d'un grand talent aussi, venait de publier les Amours des Anges et Lalla Rookh, poèmes indiens (sic). Il était alors à Paris, jouissant dans un applaudissement universel de la fleur et de la primeur de son talent. Je le voyais souvent chez Mme la duchesse de Broglie, fille de Mme de Staël, et femme dont la beauté, la vertu, l'enivrement mystique et la piété céleste devaient ravir le poète irlandais et faire croire à la saur des anges que Vigny voulait créer pour type idéal des amours sacrées...'

Moore était-il sensible en effet à la grace séraphique de Madame de Broglie? Son Journal ne témoigne, à cet égard, que d'une satisfaction assez vulgaire à la trouver très enthousiaste des Mélodies irlandaises (21 mai 1821), à lui dire des vers (3 avril 1821) ou à chanter avec elle (6 décembre 1821), et il ne nous informe pas du sujet des nombreuses ' conversations' notées chemin faisant. En tout cas, le poème The Loves of the Angels, écrit sur le continent, paraît peu de temps après le séjour de l'auteur à Paris: il est mis en vente dès janvier 1823². La même année paraissent deux traductions en prose, d'abord celle de Davésiés de Pontès ${ }^{3}$, ensuite celle de Madame Belloc ${ }^{4}$, que Moore reçoit le 15 juillet 1823, 'avec une lettre fort flatteuse... Mme Belloc dit qu'il y a deux autres personnes occupées à traduire les Anges en vers".'

Mme Belloc publiait à la suite de sa traduction celle des Mélodies irlandaises: les admirateurs français du poète possédaient ainsi dans un même volume, agrémenté d'un portrait lithographié, une version assez habile de deux cuvres bien propres à plaire au public de cette époque. Vigny savait assez d'anglais dès ce moment pour se passer d'une traduction, et peut-être prit-il connaissance des Loves of the Angels avant son départ pour Strasbourg en mars 1823. Mais c'est plutôt, à

${ }_{1}$ Cours familier de littérature, t. xvr. Paris, 1863, p. 232. Reproduit dans les Souvenirs et Portraits, t. III. Paris, 1872 , p. 146.

2 D'après Schultz-Gora, art. cité, p. 278.

3 Les Amour's des Anges, poème en III chants, trad. de l'anglais. Paris, Pillet aîné, 1823.

+Les Amours des Anges et les Mélodies irlandaises de Thomas Moore, trad. de l'anglais par Mme Louise $\mathbf{S w}=$ Belloc, traducteur des Patriarches. Paris, Chasseriau, 1823.

s Peut-être fait-elle allusion à la traduction Ducrest de Villeneuve, dont un fragment paraît dans l'Almanach des Muses de 1827, p. 80. Cf. aussi Douze mélodies françaises avec accompagnement de piano ou de harpe, paroles imitées de Th. Moore, par le C'te Auguste de Lagarde. Paris, 1823. Mme A. Tastu traduit a cette époque diverses Melodies irlandaises. Ce n'est qu'en 1829 et 1830 que devaient paraître les traductions en vers des Amours des Anges par Aroux et par Moutardier. 
mon sens, après son arrivée à Bordeaux à l'automne, et lorsqu'il se remit à travailler ce Satan qui l'avait occupé pendant l'été, que Vigny lut et relut le poème de Moore, et cela dans la traduction de Mme Belloc. Cette dernière était en rapports assez intimes avec la famille Gay $^{1}$, et Vigny, dont on sait les relations avec Mme Gay et la belle Delphine à cette époque, avait toutes les raisons d'être au courant de ses travaux. Il emprunte à sa traduction des Mélodies irlandaises le thème de l'espèce de romance-barcarolle qu'il intitule le Bateau'. Et pendant le mois où son Satan, devenant Eloa, 's'étend beaucoup sous ses doigts,' et s'augmente 'd'immenses développements', comme il l'écrit à V. Hugo le 3 octobre, il a sous la main cette traduction. Lui qui trouvait précisément à cette date que 'Lamartine a manqué son ciel comme tous ceux qui en ont fait,' il avait besoin d'étoffer sa trame et de documenter ses notions du monde angélique.

L'action proprement dite, dans Eloa, ne doit pas grand chose à l'œuvre de Moore, et l'on a raison de chercher des précédents de plus haute allure au byronisme latent dans ce poème de la Scour des Anges. M. Schultz-Gora cependant remarque justement que dans le Paradis et la Péri c'est, après deux tentatives infructueuses, grâce à l'offrande qu'elle peut faire de la larme d'un criminel repentant, que la Péri exilée du Ciel en retrouve enfin l'accès. D'autre part, quelque parallélisme épisodique ne laisse pas d'apparaître, entre l'ignorante et la pitoyable curiosité de la tendre Eloa et les amours des trois anges masculins de Moore pour des mortelles qu'ils aiment, l'un par sensualité, l'autre par un culte excessif pour les créatures de Dieu, le troisième par pure simplicité de cœur. Le deuxième ange de Moore, comme le Satan de Vigny, fonde sa séduction sur l'inconscient appel des sens :

Moore, p. 43 de la trad. Belloc:

Là, habitaient tant d'innombrables choses qui nourrissent l'ardeur des jeunes cœurs, les désirs vagues, les tendres illusions, les rêves d'amour encore sans objet, les espérances légères et ailées qui obéissent au désir..., et les passions cachées sous des pensées virginales...
Vigny, vers 427 :

Sur l'homme j'ai fondé mon empire de flamme,

Dans les désirs du cœur, dans les rêves de l'âme,

Dans les désirs du corps, attraits mystérieux,

Dans les trésors du sang, dans les regards des yeux.

Le premier ange de Moore, lorsqu'il a goûté au 'breuvage enivrant de la terre,' éprouve un peu des ivresses coupables que se rappelle l'archange déchu de Vigny:

1 Cf., sur Mme Belloc, les Souvenirs inédits de Delécluze, Revue rétrospective, 1888, t. II. pp. 22 et 195 .

${ }^{2}$ Ratisbonne la croyait inédite : elle a paru dans la Revue des Deux Mondes de 1831 et dans l'Almanach des Muses de 1832. 
Moore, p. 23 :

...remplissant [mon âme égarée] de vaines illusions, de folles pensées, et de ce désir du mal qui nous poursuit en l'absence des rayons du ciel...
Vigny, vers 666 :

Triste amour du péché! sombres désirs du mal:

De l'orgueil du savoir gigantesques pensées :

Comment ai-je connu vos ardeurs insensées?

En dépit de ces rencontres accessoires que l'analogie de quelques situations ne pouvait manquer d'amener, l'intention et la conduite de l'action offrent chez les deux poètes des divergences capitales sur lesquelles il est inutile d'insister. Mais les Amours des Anges, très documentées sur les mystères des mondes célestes et traînant à leur suite, même dans la traduction française, tout un appareil de références, permettaient à Vigny de se renseigner sur maint détail de l'existence angélique ou même de la condition physique des anges.

Moore, p. 30 :

Quoique le jour eût disparu, ses ailes diaprées étincelaient de mille feux, qu'animées de l'éclat d'Eden, elles ne tiraient que d'elles-mêmes...
Vigny, vers 631 :

Et comme, tout nourris de l'essence première,

Les anges ont au cœur des sources de lumière,

Tandis qu'elle parlait, ses ailes à l'entour,

Et son sein et son bras répandirent le jour.

Une autre irradiation éblouissante-traditionnelle, celle-ci-est celle qui émane de Dieu, et que les anges eux-mêmes ne peuvent supporter.

Moore, p. 75 :

Souvent, quand du front du TrèsHaut s'échappait un éclair trop vif pour le supporter, et que tous les Séraphins se voilaient le visage de leurs ailes, et n'osaient en contempler l'éclat...
Vigny, vers 636 :

L'archange s'en effraie, et sous ses cheveux sombres

Cherche un épais refuge à ses yeux éblouis ;

Il pense qu'à la fin des temps évanouis

Il lui faudra de même envisager son maitre,

Et qu'un regard de Dieu le brisera peut-être...

Ou, avec une image qui s'imposait à propos de splendeurs insoutenables,

Moore, p. 62 :

...apprendre à supporter cet éclat, comme les jeunes aigles supportent celui du soleil...
Vigny, vers 651 :

[l'aigle des Asturies]

Regarde son soleil, d'un bec ouvert l'aspire... 
L'émoi et le scandale seraient les mêmes, dans ces deux paradis, si l'on y évoquait le souvenir de l'Archange révolté:

$$
\text { Moore, p. } 18 \text { : }
$$

...ce feu dévorant qu'on ne nomme point aux cieux.
Vigny, vers 124 :

Nul ange n'oserait vous conter son histoire,

Nul ange n'oserait dire une fois son nom.

Semblable à la mortelle qu'aime le premier ange de Moore, Eloa n'éprouve cependant que de la tristesse, et point de colère, à connaître les crimes du réprouvé :

Moore, p. 20 :

Ce n'était point l'expression de la colère. Non...elle n'était pas irritée, mais triste. C'était un douleur aussi calme que profonde, un deuil qui ne permet point de larmes, tant l'amertume qui remplit le cœur s'y fixe et s'y glace.
Vigny, vers 126 :

Et l'on crut qu'Eloa le maudirait ; mais non,

L'effiroi n'altéra point son paisible visage...

La tristesse apparut sur sa lèvre glacée

Aussitôt qu'un malheur s'offrit à sa pensée...

Sur les fonctions 'cosmiques' des phalanges célestes, Moore fournissait des renseignements que ni Milton ni Chateaubriand ne donnaient aussi nettement, et qu'il savait appuyer de références dans ses notes.

\section{Moore, p. 14 :}

...créatures de lumière...qui, à chaque instant de la nuit et du jour, transmettent, à travers leurs innombrables légions, l'écho de sa parole lumineuse.

$$
\text { id., p. } 19 \text { : }
$$

...pourquoi mon destin ne m'a-t-il pas fait naitre esprit de cette belle étoile, habitant sa brillante sphère, pure et isolée comme tous ces êtres rayonnants...

$$
\text { et p. } 27 \text { : }
$$

Ce fut vers cette étoile lointaine que je la vis diriger son vol à travers l'espace lumineux, vers cette ile étincelante au milieu du firmament bleuâtre...
Vigny, vers 108 :

On le nommait celui qui porte la lumière ;

Car il portait l'amour et la vie en tout lieu,

Aux astres il portait tous les ordres de Dieu.

$$
\text { id., vers } 95 \text { : }
$$

Quel globe attend ses pas? quel siècle la demande?

et vers 193 :

...leur timide compagne

Etend l'aile et sourit, s'envole, et dans les airs

Cherche sa terre amie ou des astres déserts...

Dans le même ordre d'idées, il convient de remarquer que le second 'mystère' de Vigny, le Déluge qu'il écrivit cette même année 1823, conserve à l'égard des Amours des Anges une dépendance qui 
mérite d'être notée, même à côté de celle qui le rattache au Ciel et Terre de Byron. Le renvoi au $6^{\mathrm{e}}$ livre de la Genèse, qui parle des amours des anges pour les filles des hommes, se retrouvait dans la Préface de Moore. Emmanuel pourrait être le fils d'un des coupables amants dont il avait raconté les amours; et une certaine analogie de mise en scène paraît dans le début des deux poèmes:

\section{Moore, p. 13:}

Le monde était dans sa fleur; les étoiles brillantes venaient de commencer leur course radieuse... La terre était alors plus près du ciel que dans ces jours de crime et de désolation, etc.
Vigny, vers 1 du Deluge:

La terre était riante et dans sa fleur première...

Rien n'avait dans sa forme altéré la nature,

Et des monts réguliers l'immense architecture

S'élevait jusqu'aux cieux par ses degrés égaux...

C'est dans ce qu'on pourrait appeler la tonalité du décor et des accessoires que Vigny témoigne surtout qu'il a beaucoup retenu de sa lecture de Moore. De fait, il y avait là, pour un poète qui avait à dépeindre ou à suggérer les détails d'un monde transcendant, une documentation précieuse. Les 'mystères' de lord Byron, sous leur forme dramatique, n'offraient pas beaucoup de ressources à cet égard. Et il faut bien reconnaître que l'Empyrée somptueux et hiérarchique de Milton, le vaste Ciel d'oratorio évoqué par Klopstock, avaient moins chance de réveiller, chez le Vigny de 1823, des émulations fécondes que le coloris brillant—et souvent brillanté-que Moore avait donné à ses descriptions paradisiaques. On a souvent remarqué avec quelle peine Vigny s'est défait d'une certaine prédilection pour l'afféterie et la fausse élégance dans l'expression : l'ingéniosité maniérée, mais gracieuse, du barde irlandais était bien propre à satisfaire ces affinités-là, d'autant plus qu'elle correspondait à merveille aux tendances d'une époque qui n'avait pas encore rénové en matière de langue poétique et qui cherchait assez péniblement une terminologie propre à exprimer ses rêves et ses imaginations d'au-delà. 'Le vague, disait la Préface du traducteur des Amours des Anges, qui fait un des charmes de sa poésie, serait à peine toléré dans notre prose. On a beau planer dans la région des fantômes et des nuages, il faut pour nous que chaque être ait un corps et chaque objet un nom. En exprimant une pensée, Moore en éveille mille; il dessine une image, et il en fait apparaître une foule dans le lointain. Il laisse au lecteur le soin de les deviner et d'achever ses tableaux.' C'est bien ainsi que se posait la question de l'image ou de l'épithète suggestive pour cette peinture du monde céleste qu'entreprenait 
Alfred de Vigny; par là, bien plus que pour la conception ou l'agencement du poème, la Sour des Anges est tributaire des Amours des Anges: et si les emprunts ou les réminiscences se réduisent à des touches de couleur, il n'en reste pas moins que la tonalité générale du tableau s'en trouve déterminée. Grâce à une qualité bien plus haute d'esprit et d'âme, grâce à d'autres modèles infiniment plus forts, Vigny rehausse souvent d'un ton plus ferme, accentue d'un trait mieux cerné un détail qui a son analogue chez Moore; et il suffit de juxtaposer des exemples comme ceux-ci pour faire valoir l'avantage d'Eloa:

$$
\text { Moore, p. } 34 \text { : }
$$

Dès l'instant où je fus appelé avec les chérubins pour assister au premier réveil printanier de la nature dans ces sphères florissantes, ces fleurs lumineuses qui jaillirent au premier souffle de l'Eternel...

id., p. 36 :

...de nouveaux mondes, brillants de jeunesse et de fraîcheur, semblaient s'élancer du sein des ténèbres...

...Celui qui venait de parcourir cette vaste étendue où étincellent des mondes entassés...
Vigny, vers 153 :

Et soit lorsque Dieu même, appelant les esprits,

Dévoilait sa grandeur à leurs regards surpris,

Et montrait dans les cieux, foyer de la naissance,

Les profondeurs sans nom de sa triple puissance...

vers 88 :

Et des fleurs qu'au Ciel seul fit germer la nature...

$$
\text { id., vers } 763 \text { : }
$$

Des anges au Chaos allaient puiser des mondes.

Passant avec terreur dans ses plaines profondes...

Mais ailleurs, le poète d'Eloa s'en tient à la qualité même de la description ou de l'évocation tentée par Moore. La lumière d'Eden, chez l'un et chez l'autre, est plutôt azurée ou nacrée que franchement éclatante; une certaine mollesse asiatique semble s'insinuer dans leur imagination, et l'on est loin, au milieu des fleurs, des fontaines au sable vermeil, des météores indistincts et des arcs-en-ciel flottants de ces paradis en demi-teintes, du Ciel puritain de Milton et du Ciel évangélique de Klopstock. Comment les anges n'y prendraient-ils pas le goût de voluptés moins célestes? Une sorte de suave frivolité n'en est point bannie.

$$
\text { Moore, p. } 38 \text { : }
$$

J'avais vu naitre la première femme, Eve... J'avais vu les anges les plus purs s'incliner au-dessus d'elle en l'adorant.
Vigny, vers 80 :

Et tous les Anges purs, et tous les grands Archanges

Abaissèrent leur front jusqu'à ses pieds de neige... 
Les comètes et les météores, dans ce firmament peu rigide, semblent presque l'emporter sur les étoiles fixes:

Moore, p. 36 :

...je suivais quelque comète royageuse se dirigeant au loin vers des points lumineux.
Vigny, vers 308 :

Chaque étoile semblait poursuivre un météore ;

Et l'ange en souriant au spectacle étranger,

Suivait des yeux leur vol circulaire et léger.

$$
\text { id., p. } 72 \text { : }
$$

Elle s'était évanouie, comme un météore qui luit tout-à-coup sur nos têtes, et qui s'enfuit au moment où l'on crie : 'Voyez, voyez !...'

$$
\text { id., vers } 57 \text { : }
$$

Comme on voit la comète errante dans les cieux

Fondre au sein de la nuit ses rayons glorieux.

Les deux poètes se servent de la même expression pour désigner le mouvement des astres entraînés dans le mouvement de l'univers :

$$
\text { Moore, p. } 35 \text { : }
$$

...les astres,...roulant au milieu de l'espace comme des chars vivants de lumière ${ }^{1} \ldots$
Vigny, vers 144 :

Chars vivants dont les yeux ont d'éclatants prestiges !

Même analogie dans quelques-uns des jeux auxquels les anges se livrent avec les astres:

$$
\text { Moore, p. } 35 \text { : }
$$

...je parcourais soir et matin les lignes radieuses qui s'étendent comme des réseaux d'or entre les étoiles et le soleil, déliant tous ces rayons de lumière...
Vigny, vers 577 :

$\mathrm{Du}$ char des astres purs j'obscurcis les essieux,

Je voilai leurs rayons pour attirer tes yeux...

Sur terre, ou à la surface du chaos, rôdent des feux follets semblables:

$$
\text { Moore, p. } 23 \text { : }
$$

...les feux livides qui rampent à la surface de la terre dès que le jour i disparu.
Vigny, vers 305 (cf. vers 471):

Mais elle y vit bientôt des feux errants et bleus

Tels que des froids marais les éclairs onduleux.

1 M. Schultz-Gora, art. cité, p. 283, rapporte les 'chars vivants' de Vigny à des passages miltoniens fort peu convaincants. C'est le lien de signaler quelques errata qu'appelle son travail. L'influence de Heaven and Earth sur le Déluge de Vigny avait été indiquée expressément par M. E. Dupuy, art. cité, p. 406 (dans la Jeunesse des Romantiques, p. 353); M. Schultz-Gora (p. 281) a lu pensée régénératrice où M. Dupuy avait écrit pensée génératrice, ce qui change sensiblement la thèse; lire (p. 283) VI au lieu de V pour la seconde indication du Paradis perdu. 
Nous voici dans une partie de la création plus accessible et mieux connue; nombre de détails qui lui sont attribués se retrouvent chez les deux poètes. La musique sur la mer:

\section{Moore, p. 76 :}

Ce fut pendant le crépuscule du soir, sur le rivage de la mer tranquille, qu'il entendit pour la première fois les sons du luth et la voix de celle qu'il aima glisser sur les eaux argentées...

La jeune épouse :

\section{Moore, p. 19 :}

...comme la jeune épouse qui se penche sur le bord du lit nuptial...

Le ver luisant:

Moore, p. 62 :

La lumière que le ver luisant suspend la nuit aux branches des arbres...

\section{Les amours des fleurs:}

$$
\text { Moore, p. } 63 \text { : }
$$

...la rose, confiante et sans tache, qui a reçu toute la nuit les baisers de la mouche de feu?

Le serpent-oiseleur:

Moore, p. 14 :

...semblable à l'oiseau qui abandonne son nid élevé, fasciné par des yeux séducteurs...

Les diamants dans l'obscurité :

$$
\text { Moore, p. } 51 \text { : }
$$

..les diamants, semblables à des yeux qui brillent au milieu des ténèbres, furent surpris dans leur retraite obscure...
Vigny, vers 615 :

Et la mer quand ses flots apportent sur la grève

Les chants du soir aux pieds du voyageur qui rêve...

Elle marche vers Dieu comme une épouse au temple...

Vigny, vers 469 :

Le vermisseau reluit; son front de diamant

Répète auprès des fleurs les feux du firmament...

Vigny, vers 436 :

Comme le papillon, sur ses ailes poudreuses,

Porte aux gazons émus des peuplades de fleurs

Et leur fait des amours sans périls et sans pleurs.

Vigny, vers 214 :

Les serpents-oiseleurs qu'elles pourraient cacher.

et vers 423 :

Sous l'éclair d'un regard sa force fut brisée ;

Et dès qu'il vit ployer son aile mấtrisée,

L'ennerni séducteur ${ }^{1} \ldots$

Vigny, vers 635 :

Ainsi le diamant luit au milieu des ombres.

L'archange s'en effraie, et sous ses cheveux sombres

Cherche un épais refuge à ses yeux éblouis...

1 Il faut noter que le passage d'Atala qui-la remarque en a été souvent faite-a fourni la plupart de ses détails à l'épisode du colibri des Florides, mentionne le serpent-oiseleur sans le montrer dans l'exercice de son pouvoir de fascination. 
La jeune étoile-cette épithète de jeune est assez particulière:

Moore, p. 48 :

Les nuages d'automne qui retiennent les éclairs prêts à s'échapper de leurs flancs, pour laisser briller une jeune étoile.
Vigny, vers 555 :

Toi seule m'apparus comme une jeune étoile

Qui de la vaste nuit perce à l'écart le voile.

(Cf. deux 'jeunes planètes' au vers 223.)

Un autre détail est un souvenir du Paradis et la Péri:

Moore, vers 167 :

Vigny, vers 605 :

Ces lis vierges qui baignent toute la Son bras, comme un lis blanc sur le lac nuit leur beauté dans le lac... suspendu...

Il serait possible de continuer ces rapprochements. Quelques détails d'un orientalisme assez choquant dans Eloa-les 'Divans où dort la molle Asie' et toute cette attitude de jeune satrape de l'ange déchu (vers 353 et suivants), la blanche tour d'Alep et sa sultane imprévue (v. 420)—ont peut-être leur origine dans l'exotisme de LallaRookh, moins éclatant et plus insinuant que celui de Byron. Vigny, qui a toujours eu, pour les effets de lumière contrariée et d'ombre transparente, une prédilection dont témoignent presque tous les tableaux lumineux de son œuvre, trouvait un coloriste à sa guise dans le poète des Amours des Anges: Moore ne pousse-t-il pas le raffinement jusqu'à ébaucher 'l'arc-en-ciel formé par la lune'! C'est par là, par cette délicatesse maniérée dans le coloris, qu'il a dû séduire le jeune officier à la pensée si grave qui devait conserver longtemps encore, malgré toutes les hardiesses de sa méditation, le goût un peu mièvre du 'joli' et de l'ingénieux dans l'expression.

Fernand BaLdensPerger. 